\title{
Incidencia de las técnicas de gestión en la mejora de las decisiones administrativas
}

\section{Impact of management techniques on the improvement of administrative decisions}

\author{
Dayana Valdés Pérez* \\ dvaldes@pucem.edu.ec
}

\begin{abstract}
Resumen
Demostrar la utilidad del uso de técnicas de gestión para la toma de decisiones gerenciales, constituyó el objetivo de esta propuesta, que fue validada a través de un estudio de caso aplicado a la línea de producción de jugos de frutas de una empresa industrial cubana, escogida como muestra intencional. Se formuló un procedimiento para el análisis de las variables costos, gastos, punto de equilibrio, margen de seguridad, apalancamiento operativo y beneficio, así como la valoración de dos escenarios que permitieron diagnosticar la situación de la industria, proyectar niveles acertados de beneficios, indicando que los resultados poco alentadores de la empresa en parte se debieron a la limitada utilización de técnicas de gestión como apoyo a los procesos administrativos.
\end{abstract}

\section{Palabras clave}

Apalancamiento operativo, margen de seguridad, punto de equilibrio, rentabilidad.

* Cubana, Doctora en Ciencias Contables y Financieras. Pontificia Universidad Católica Ecuador sede Manabí. Directora de Investigación, Publicaciones y Postgrado. 


\section{Abstract}

Demonstrate the usefulness of the use of management for management decision-making techniques, it was the objective of this proposal, which was validated through a case study applied to the production line of fruit juices from a Cuban industrial company, chosen as intentional sample. It was formulated as a procedure for the analysis of the costs variable, expenses, point of balance, margin of safety, leverage operating and benefit, as well as the valuation of two scenarios that allowed diagnose the situation of the industry, project levels successful of benefits, indicating that the little results of the company was caused, by partly, by the limited use of technical of management as support to them processes administrative.

\section{Keywords}

Balance point, margin of safety, operating leverage, profitability.

Forma sugerida de citar: Valdés Pérez, Dayana (2016). Incidencia de las técnicas de gestión en la mejora de las decisiones administrativas. Retos, 12(2), pp. 199-213.

\section{Introducción}

El perfeccionamiento continuo de los procesos ha potenciado el desarrollo de técnicas de creación y transformación de escenarios estratégicos y económicos, convenientes para el beneficio de la propia organización. Con el transcurrir de los años los productos y servicios son cada vez más complejos y con ello las exigencias de los más diversos clientes, en cuanto a calidad, tiempo, diseño y precio. Ello, junto con la presencia de mercados cada vez más globalizados hace que las empresas se muevan en medios muy competitivos y que para poder triunfar tengan que lograr una adecuada gestión de cualquiera de las áreas de su entidad siendo importante, además, una correcta relación entre ellas (Pastrana, 2012). En la actualidad Cuba se ha convertido en un país mayormente importador y en tales circunstancias, teniendo en cuenta las dificultades por la que atraviesa la isla, la economía está orientada hacia su total recuperación, para ello la elevación de la eficiencia y la productividad deberá convertirse en la vía principal para asegurar el desarrollo económico. Para lograr esto es necesario convertir a las empresas en organizaciones rentables y fomentar el perfeccionamiento de las ventajas competitivas de dichas entidades, Gómez et al. (2012), entre 
los factores más reconocidos como fuente de ventaja competitiva se encuentran los recursos comerciales, por tanto la correcta orientación de los procesos juega un rol principal.

La rama industrial ocupa un lugar preponderante dentro de la estructura económica nacional. Sin embargo, las empresas pertenecientes a este sector están inmersas en este contexto económico tan complejo, lo cual se torna preocupante por el posicionamiento que las producciones industriales tienen en el mercado internacional, así como la infraestructura tecnológica con que se cuenta para obtener niveles óptimos de eficiencia, aun cuando se infieren algunos elementos asociados al control económico, no está suficientemente fundamentada la necesidad de asumir en las empresas criterios de información donde la utilización de herramientas de la contabilidad de gestión constituya un elemento preponderante para facilitar no solo el registro de las operaciones sino también su control y toma de decisiones oportunas por parte de la dirección.

Sinisterra (2011), plantea que hasta 1980 las empresas industriales consideraban que sus procedimientos de acumulación de costos constituían secretos industriales, pues el sistema de información financiera no incluían las bases de datos y los archivos, esto se tradujo en un estancamiento con relación a otras ramas de la contabilidad hasta que se comprobó que su aplicación producía beneficios.

Todo esto favoreció el predominio de criterios empíricos en la toma de decisiones que ha originado el desaprovechamiento de capacidades generadoras de ingresos, retardando la recuperación de importantes inversiones. Se ha prestado mayor atención a la restricción de costos y gastos que a la elevación de la calidad y del valor agregado de las producciones, dando lugar a un deterioro progresivo del producto y limitados resultados en la comercialización.

La apertura de Cuba a las relaciones económicas internacionales se ha dirigido a sectores y ramas importantes, entre los cuales se encuentra la producción industrial, la que ha devenido en una significativa fuente de ingresos de divisas al país y cumple con una destacada función dentro de la economía interna. Uno de los aspectos clave para lograr su desarrollo, es la elevación de la eficiencia empresarial, con el fin de mejorar la gestión administrativa de la empresa que según lo manifestado por (Culqui, 2012, p. 29) funciona en base a la determinación y la satisfacción de muchos de los objetivos en los aspectos políticos, sociales y eco- 
nómicos que reposan en la competencia que posea el administrador. El fin de la persona responsable de la gestión administrativa es garantizar la disposición de determinadas capacidades y habilidades con respecto a este tema y perfeccionar su entorno financiero.

Esto nos conduce a resaltar la importancia de la gestión administrativa que según lo indicado por Torres (2010) citado por (Villalta, 2011, p. 65): "Para consolidar dicho proceso, las empresas deben emprender un conjunto de acciones destinadas a obtener una Administración más transparente y con mayor capacidad de gestión, centrada en la mejora de la economía, la eficacia y la eficiencia de la actividad administrativa, en el desarrollo de las capacidades directivas, en el cambio de cultura administrativa”... elementos que han quedado un tanto excluidos en los procesos de gestión actual.

La problemática vigente a resolver en el corto plazo radica en la insuficiente utilización por parte de las empresas industriales de instrumentos, métodos, herramientas que generen información fidedigna para ser empleada por la gerencia en sus procesos y toma de decisiones.

Un diagnóstico realizado en las industrias ubicadas en el centro del país, sustentado en el análisis documental, la consulta con espe- cialistas del sector y la elaboración y estudio de series cronológicas, que mostraron una tendencia al incremento de los costos, así como bajos rendimientos, evidenció que estas organizaciones han operado con limitados niveles de eficiencia y rentabilidad y las decisiones administrativas se veían frenadas en ocasiones por falta de información relevante.

Ante esta situación la investigación responde a las siguientes interrogantes: ¿Cómo utilizar las técnicas de gestión para la mejora de los proceso de planificación, control y toma de decisiones? ¿Cómo contribuyen las técnicas de gestión de costos a elevar los niveles de comercialización de las empresas industriales?

\section{Metodología}

(Herrscher, 2010), como una respuesta a la situación creada por las exigencias del nuevo entorno a las empresas, han surgido las nuevas tecnologías de gestión, procedimientos desarrollados para optimizar el uso de los recursos del ente para alcanzar un objetivo y que tienen incidencia en los procedimientos y en la información que emana de los Sistemas de Contabilidad de Gestión, Salgado (2011) cuando se va a tomar cierto tipo de decisiones, especialmente en las empresas industriales, la contabilidad financie- 
ra no proporciona la información suficiente y necesaria, por lo que resulta imprescindible buscar las vías para alcanzar mejores resultados.

Gestionar de forma anticipada constituye actualmente un imperativo de las organizaciones que transitan el camino a la excelencia, predecir anticipadamente la forma en que se desempeñarán sin dudas minimiza el riesgo de las operaciones y establece una garantía en el cumplimiento de una función básica como es la planificación, donde el uso de modelos y técnicas de gestión que devienen de su aplicación a partir de la información contable de la entidad constituye el punto de partida de la investigación, que formula un estudio de caso, tomando como referencia una empresa dedicada al procesamiento y comercialización de frutas y vegetales, escogida como muestra intencional.

La empresa industrial muestra está ubicada en el centro de Cuba, en la provincia de Ciego de Ávila. La misma cuenta con varias líneas de producción, basadas fundamentalmente en el procesamiento de cítricos y frutas tropicales, con el objetivo de abastecer el mercado internacional y nacional (principalmente a los hoteles de todo el país). Dentro de las principales líneas de producción de la entidad se encuentra la elaboración y el procesamiento de:
- Jugos concentrados congelados de cítricos y piña

- Puré de frutas (tanto congelado como aséptico)

- Néctares asépticos (diferentes sabores)

- Tomate y enlatado del mismo

- Mermeladas

- Papas pre fritas

Para el proceso de planeación se tomó como base la información contable contenida en los estados financieros de la empresa considerando no sólo las condiciones que generan cambios en los niveles de actividad, sino también los resultados históricos medios, o sea ni los mejores, ni los peores, una vez consideradas estas valoraciones, se toma como año estándar el 2014 y sus resultados permitieron realizar la planeación futura para la línea de producción de jugos, por ser la que mayores ingresos le proporciona a la industria. Partiendo de que en esta línea se elaboran diferentes tipos de jugos con similares características pero con gastos y precios diferenciados se clasificaron las partidas de costos y gastos en su relación con el volumen de actividad en fijos y variables lo que facilitó el cálculo de las diferentes variables a considerar en el proceso de gestión contable para la toma de decisiones como son: el punto de equilibrio, margen de seguridad, rentabilidad, fluctua- 
ciones en las ventas, utilidades y grado de apalancamiento operativo (GAO).

Fueron empleados métodos del nivel empírico, entre los que se destacan, la entrevista y la consulta con expertos. La entrevista estructurada, con el objetivo de delimitar los procesos y actividades que se desarrollan en la empresa, se realizó para todos los directivos y mandos intermedios y la opinión de los especialistas de la subdirección de calidad y economía.

De forma general se aplicó el método histórico-lógico, se recopiló y procesó información correspondiente al año 2014, para evaluar y analizar la situación presentada por la empresa en este período y poder realizar la proyección de periodos posteriores y su diagnóstico.

La investigación partió de lo general, el estudio de la empresa a lo particular, el comportamiento de sus indicadores de resultados y más específico, el costo, permitiendo realizar proyecciones a partir de la valoración de escenarios donde se estimaron cambios de acuerdo a las condiciones reales y estudios realizados del posicionamiento en el mercado de los productos, contribuyendo a la adecuada planeación y toma de decisiones por parte de la administración.

\section{Resultados}

Se presentan los gastos correspondientes y su clasificación en fijos y variables en la tabla 1.

\section{Tabla 1. Clasificación de gastos y precio de los productos para la línea jugos de frutas naturales}

\begin{tabular}{|l|l|l|l|}
\hline \multicolumn{1}{|c|}{ Producciones } & \multicolumn{1}{|c|}{$\begin{array}{c}\text { Gastos } \\
\text { variables }\end{array}$} & \multicolumn{1}{|c|}{ Gastos fijos } & Precio \\
\hline Néctar Mango & $\$ 75.00$ & $\$ 1173.00$ & $\$ 88.52$ \\
\hline Néctar Guayaba & 77.43 & 1173.00 & 82.52 \\
\hline Néctar Naranja-Mango & 76.87 & 1173.00 & 89.95 \\
\hline Néctar Naranja-Piña & 64.62 & 1173.00 & 77.71 \\
\hline Néctar Naranja-Plátano & 70.75 & 1173.00 & 83.83 \\
\hline Néctar Papaya & 75.34 & 1173.00 & 87.42 \\
\hline Cóctel de frutas & 89.68 & 1173.00 & 92.76 \\
\hline Naranja Fruta Bomba & 75.00 & 1173.00 & 86.08 \\
\hline
\end{tabular}

Fuente: Elaboración de la autora a partir de la información de la empresa. 
El cálculo del punto de equilibrio se muestra en la tabla 2 en unidades y en pesos.

Tabla 2. Punto de equilibrio en unidades y en pesos (PE)

\begin{tabular}{|l|l|l|}
\hline \multicolumn{1}{|c|}{ Producciones } & \multicolumn{1}{|c|}{ PE en unidades } & \multicolumn{1}{|c|}{ PE en pesos } \\
\hline Néctar Mango & 87 & $\$ 7701.24$ \\
\hline Néctar Guayaba & 230 & 18979.6 \\
\hline Néctar Naranja-Mango & 90 & 8095.5 \\
\hline Néctar Naranja-Piña & 90 & 6993.9 \\
\hline Néctar Naranja-Plátano & 90 & 7544.7 \\
\hline Néctar Papaya & 97 & 8479.74 \\
\hline Cóctel de frutas & 380 & 35248.8 \\
\hline Naranja Fruta Bomba & 106 & 9188.08 \\
\hline
\end{tabular}

Fuente: Elaboración propia

El Margen de Seguridad (MS) el año 2015, los resultados de su cálse calcula para cada producto para culo se muestran en la tabla 3.

\section{Tabla 3. Resultados del cálculo del Margen de seguridad}

\begin{tabular}{|l|l|l|l|}
\hline \multicolumn{1}{|c|}{ Producciones } & \multicolumn{1}{|c|}{$\begin{array}{c}\text { Ventas esperadas } \\
(\mathrm{u})\end{array}$} & Ventas en PE $(\mathrm{u})$ & $\begin{array}{l}\text { \% Margen de } \\
\text { seguridad }\end{array}$ \\
\hline Néctar Mango & 8700 & 87 & 99 \\
\hline Néctar Guayaba & 4300 & 230 & 95 \\
\hline $\begin{array}{l}\text { Néctar Naranja- } \\
\text { Mango }\end{array}$ & 9000 & 90 & 99 \\
\hline Néctar Naranja-Piña & 9500 & 90 & 99 \\
\hline $\begin{array}{l}\text { Néctar Naranja- } \\
\text { Plátano }\end{array}$ & 9850 & 90 & 99 \\
\hline Néctar Papaya & 9700 & 97 & 99 \\
\hline Cóctel de frutas & 3800 & 380 & 90 \\
\hline Naranja Fruta Bomba & 1060 & 106 & 90 \\
\hline
\end{tabular}

Fuente: Elaboración propia 
El cambio de las utilidades y la tas por producciones según se muesrentabilidad cuando varían las ven- tra en las tablas 4 y 5 .

Tabla 4. Resultados del cálculo de los beneficios por producciones

\begin{tabular}{|l|l|l|l|}
\hline \multicolumn{5}{|c|}{ Beneficio = ingresos - costos } \\
\hline \multicolumn{1}{|c|}{ Producciones } & \multicolumn{1}{|c|}{ Beneficio } & \multicolumn{1}{|c|}{ Ingresos } & \multicolumn{1}{|c|}{ Costos } \\
\hline Néctar Mango & $\$ 779.92$ & $\$ 2168.44$ & $\$ 1388.52$ \\
\hline Néctar Guayaba & 779.91 & 2150.43 & 1370.52 \\
\hline Néctar Naranja-Mango & 779.92 & 2149.87 & 1369.95 \\
\hline Néctar Naranja-Piña & 779.91 & 2407.62 & 1627.71 \\
\hline Néctar Naranja-Plátano & 779.92 & 2343.75 & 1563.83 \\
\hline Néctar Papaya & 779.92 & 3448.34 & 2668.42 \\
\hline Cóctel de frutas & 779.92 & 3872.68 & 3092.76 \\
\hline Naranja Fruta Bomba & 779.92 & 3126.00 & 2346.08 \\
\hline
\end{tabular}

Fuente: Elaboración propia

Tabla 5 Resultados del cálculo de la rentabilidad por producciones

\begin{tabular}{|l|l|}
\hline \multicolumn{1}{|c|}{ Producciones } & \multicolumn{1}{|c|}{$\%$ de Rentabilidad } \\
\hline Néctar Mango & 56.17 \\
\hline Néctar Guayaba & 56.91 \\
\hline Néctar Naranja-Mango & 56.93 \\
\hline Néctar Naranja-Piña & 47.91 \\
\hline Néctar Naranja-Plátano & 49.87 \\
\hline Néctar Papaya & 9.23 \\
\hline Cóctel de frutas & 25.22 \\
\hline Naranja Fruta Bomba & 33.24 \\
\hline
\end{tabular}

Fuente: Elaboración propia

La comparación de las variables calculadas anteriormente se muestra en la tabla 6. 
Tabla 6. Comparación entre el Margen de seguridad, Rentabilidad y

GAO por producciones

\begin{tabular}{|l|l|l|l|l|l|}
\hline \multicolumn{1}{|c|}{ Producciones } & \multicolumn{1}{|c|}{ Ventas } & Beneficio & Rentabilidad & MS & GAO \\
\hline Néctar Mango & $\$ 2168.44$ & $\$ 779.92$ & 35.97 & 99 & 0.1 \\
\hline Néctar Guayaba & 2150.43 & 779.91 & 36.27 & 95 & 0.2 \\
\hline $\begin{array}{l}\text { Néctar Naranja- } \\
\text { Mango }\end{array}$ & 2149.87 & 779.92 & 36.28 & 99 & 0.1 \\
\hline $\begin{array}{l}\text { Néctar Naranja- } \\
\text { Piña }\end{array}$ & 2407.62 & 779.91 & 32.39 & 99 & 0.1 \\
\hline $\begin{array}{l}\text { Néctar Naranja- } \\
\text { Plátano }\end{array}$ & 2343.75 & 779.92 & 33.28 & 99 & 0.1 \\
\hline Néctar Papaya & 3448.34 & 779.92 & 22.62 & 99 & 0.1 \\
\hline Cóctel de frutas & 3872.68 & 779.92 & 20.14 & 90 & 0.2 \\
\hline $\begin{array}{l}\text { Naranja Fruta } \\
\text { Bomba }\end{array}$ & 3126.00 & 779.92 & 24.95 & 90 & 0.9 \\
\hline
\end{tabular}

Fuente: Elaboración propia

Para prever resultados positivos se evalúan diferentes alternativas de ingresos, costos y gastos para definir la variante más óptima, en la cual se alcance una cifra máxima de utilidades o mínima de pérdidas sin deterioro de los estándares de calidad. Se introduce su demostración práctica con el fin de facilitar la interpretación de los resultados e ilustrar las ventajas que ofrece este método. Para ello se trabaja con dos escenarios como se muestra a continuación:
El primer escenario se diseña teniendo en cuenta la variabilidad en la demanda de jugos y su planificación empírica, lo que provoca que los niveles de ventas proyectados se encuentren muy por debajo de la demanda y capacidad de producción de la línea, por ello, se procede en este primer momento a incrementar en un $5 \%$ el volumen de las ventas, lo que conlleva a un aumento en los costos variables en esa misma proporción. Como se aprecia en la tabla 7 . 
Tabla 7 Resultados del Escenario 1

\begin{tabular}{|c|c|c|c|c|c|c|c|c|c|c|}
\hline \multirow[b]{2}{*}{ 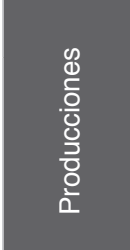 } & \multicolumn{5}{|c|}{ Actual } & \multicolumn{5}{|c|}{ Escenario 1} \\
\hline & $\underset{\square}{\amalg}$ & $\begin{array}{l}\frac{0}{0} \\
\frac{0}{\Phi} \\
\frac{0}{\Phi} \\
\infty\end{array}$ & 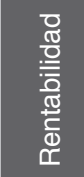 & $\sum^{\infty}$ & @্ড & $\underset{\square}{ш}$ & $\sum^{\infty}$ & $\begin{array}{l}\frac{0}{0} \\
\frac{0}{\Phi} \\
\frac{0}{\Phi} \\
\infty\end{array}$ & 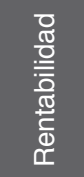 & ঠ্ণ \\
\hline $\begin{array}{l}\text { Néctar } \\
\text { Mango }\end{array}$ & 87 & $\$ 779.92$ & 35.97 & 99 & 0.1 & 120 & 98.69 & $\$ 88075.95$ & 10.89 & 0.11 \\
\hline $\begin{array}{l}\text { Néctar } \\
\text { Guayaba }\end{array}$ & 230 & 779.91 & 36.27 & 95 & 0.2 & 963 & 78.68 & 4328.53 & 1.16 & 0.01 \\
\hline $\begin{array}{l}\text { Néctar } \\
\text { Naranja- } \\
\text { Mango }\end{array}$ & 90 & 779.92 & 36.28 & 99 & 0.1 & 127 & 98.66 & 86111.92 & 10.13 & 0.10 \\
\hline $\begin{array}{l}\text { Néctar } \\
\text { Naranja- } \\
\text { Piña }\end{array}$ & 90 & 779.91 & 32.39 & 99 & 0.1 & 119 & 98.81 & 97170.52 & 12.54 & 0.13 \\
\hline $\begin{array}{l}\text { Néctar } \\
\text { Naranja- } \\
\text { Plátano }\end{array}$ & 90 & 779.92 & 33.28 & 99 & 0.1 & 123 & 98.81 & 97520.31 & 11.25 & 0.11 \\
\hline $\begin{array}{l}\text { Néctar } \\
\text { Papaya }\end{array}$ & 97 & 779.92 & 22.62 & 99 & 0.1 & 141 & 98.61 & 83494.90 & 9.38 & 0.09 \\
\hline $\begin{array}{l}\text { Coctel } \\
\text { de frutas }\end{array}$ & 380 & 779.92 & 20.14 & 90 & 0.2 & 3,011 & 23.08 & 351.89 & 0.10 & 1.00 \\
\hline $\begin{array}{l}\text { Naranja } \\
\text { Fruta } \\
\text { Bomba }\end{array}$ & 106 & 779.92 & 24.95 & 90 & 0.9 & 160 & 85.62 & 6985.29 & 7.29 & 0.07 \\
\hline
\end{tabular}

Fuente: Elaboración propia

En el segundo escenario se tuvo en cuenta la continua aceptación del producto y por tanto, el progresivo incremento de la demanda y de la capacidad de producción, por lo que se propone un aumento de los precios en un 5\%, considerando que con ello no se afecta la comercialización de la línea de jugos naturales. Como se aprecia en la tabla 8. 
Tabla 8. Resultados del escenario 2

\begin{tabular}{|c|c|c|c|c|c|c|c|c|c|c|}
\hline \multirow[b]{2}{*}{ 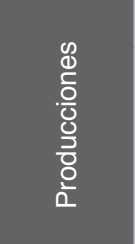 } & \multicolumn{5}{|c|}{ Actual } & \multicolumn{5}{|c|}{ Escenario 2} \\
\hline & Ш & 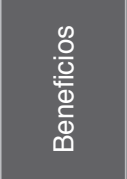 & 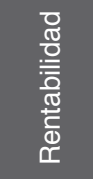 & $\sum^{\infty}$ & 워 & Ш & $\sum^{\infty}$ & $\begin{array}{l}\mathscr{0} \\
\frac{0}{0} \\
\frac{0}{\Phi} \\
\frac{0}{\Phi} \\
\infty\end{array}$ & 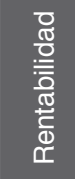 & 원 \\
\hline $\begin{array}{l}\text { Néctar } \\
\text { Mango }\end{array}$ & 87 & $\$ 779.92$ & 35.97 & 99 & 0.1 & 77 & 99 & $\$ 807241.68$ & 23.7 & 0.19 \\
\hline $\begin{array}{l}\text { Néctar } \\
\text { Guayaba }\end{array}$ & 230 & 779.91 & 36.27 & 95 & 0.2 & 149 & 97 & 371207.28 & 10.3 & 0.10 \\
\hline $\begin{array}{l}\text { Néctar } \\
\text { Naranja- } \\
\text { Mango }\end{array}$ & 90 & 779.92 & 36.28 & 99 & 0.1 & 78 & 99 & 848657.55 & 18.5 & 0.18 \\
\hline $\begin{array}{l}\text { Néctar } \\
\text { Naranja- } \\
\text { Piña }\end{array}$ & 90 & 779.91 & 32.39 & 99 & 0.1 & 96 & 99 & 773529.54 & 20.7 & 0.21 \\
\hline $\begin{array}{l}\text { Néctar } \\
\text { Naranja- } \\
\text { Plátano }\end{array}$ & 90 & 779.92 & 33.28 & 99 & 0.1 & 91 & 99 & 865447.95 & 19.5 & 0.19 \\
\hline $\begin{array}{l}\text { Néctar } \\
\text { Papaya }\end{array}$ & 97 & 779.92 & 22.62 & 99 & 0.1 & 162 & 98 & 887704.28 & 17.8 & 0.18 \\
\hline $\begin{array}{l}\text { Coctel de } \\
\text { frutas }\end{array}$ & 380 & 779.92 & 20.14 & 90 & 0.2 & 401 & 89 & 367019.64 & 7.6 & 0.08 \\
\hline $\begin{array}{l}\text { Naranja } \\
\text { Fruta } \\
\text { Bomba }\end{array}$ & 106 & 779.92 & 24.95 & 90 & 0.9 & 153 & 86 & 93460.96 & 15.8 & 0.16 \\
\hline
\end{tabular}

Fuente: Elaboración propia

\section{Discusión}

Pérez y Aspiolea (2011) señalan que la técnica de punto de equilibrio es uno de los métodos cuantitativos utilizado por los directivos, gerentes y personal vinculado a la toma de decisiones, sin embargo los mismos no deben ser absolutizados, ya que esto conduce indiscutiblemente a errores, por ello la decisión más acertada es aquella que vincula los resultados cuantitativos, resultantes de modelos, técnicas o métodos matemáticos, con el análisis cualitativo, el cual aporta conocimientos sobre el fenómeno estudiado, siempre con determinado grado de subjetividad y empirismo, cuando solo se tiene en cuenta este último.

Fue por ello que con el procedimiento propuesto se pudieron analizar indicadores como el apalancamiento operativo y la renta- 
bilidad (Suárez y Rodríguez, 2011) siempre van juntos, a mayor rentabilidad mayor apalancamiento. Entre riesgo y seguridad no existe contradicción, pues están asociados al nivel de ventas proyectado respecto a estas en punto de equilibrio.

Este es el efecto del apalancamiento operativo que ocurre como consecuencia de la existencia de gastos fijos, que no varían al incrementarse las ventas y por lo tanto los beneficios crecen más que estas. Polimeni et al. (2011), lo anterior puede parecer perfecto, sin embargo, no debe olvidarse el riesgo, que crece proporcionalmente al nivel de apalancamiento y se manifiesta de dos maneras:

1. En la elevación del punto de equilibrio según se incrementen los gastos fijos.

2. Si el pronóstico de ventas fue muy optimista y estas disminuyen en lugar de incrementarse los beneficios disminuirán mucho más que ellas.

Relacionado con lo planteado anteriormente el análisis de los resultados que se muestran en la tabla 6 , permite inferir que las producciones presentan un alto margen de seguridad, por tanto los beneficios son menores, la Rentabilidad y el Grado de apalancamiento son muy bajos, en todos los casos no alcanzan los parámetros que según la teoría deben lograr estos indicadores que demuestren una adecuada planificación de los recursos y proyección de las ventas.

En el análisis de los resultados obtenidos en los escenarios anteriores se pudo constatar que el incremento de los costos variables provoca un aumento en el punto de equilibrio y como resultado del aumento de las ventas se elevan los niveles de beneficios. Por otro lado en el análisis del riesgo operacional se concluye que el margen de seguridad es alto por tanto la rentabilidad disminuye con respecto a la situación actual y el grado de apalancamiento operativo es ínfimo, lo que indica que la entidad está trabajando en esta línea de producción con poco riesgo y no explota al máximo su capacidad.

En el escenario 2 la situación con un incremento de los precios no varía notablemente con relación al anterior, solo se constata una disminución del Punto de Equilibrio. Todo lo anterior permite afirmar que las decisiones relacionadas con la planificación de la producción afectan la comercialización y resultados de producción de la línea de jugos naturales al no aprovechar al máximo las capacidades y la demanda del mercado, además, la concatenación de un precio promedio discreto con un bajo nivel de 
ventas genera un nivel de ingresos insuficientes con respecto al volumen de gastos fijos derivados de las operaciones de la entidad.

En estudios similares, (Rendón, 2012; Arnaiz, 2012; Pérez, 2015) en empresas del turismo se puede corroborar la importancia y resultados positivos de la aplicación de herramientas de gestión de costos para la mejora en la información y toma de decisiones administrativas demostrando la elevación de los indicadores económicos, que aplicado en empresas industriales demuestran los mismos resultados favorables para la administración.

Esto nos conduce a aseverar lo expuesto por Fontalvo et al. (2012) la evaluación de indicadores como técnica administrativa ayuda a mejorar la gestión porque produce la información necesaria para identificar y entender las causas de los logros y los fracasos o problemas del desempeño individual y colectivo dentro de un contexto de planeación estratégica. Esta evaluación saca a la luz información real sobre el comportamiento del sistema objeto de estudio, que hace posible la creación de estrategias y herramientas de apoyo para la toma de decisiones que contribuyan a mejorar la estructura del mismo. Nociones como estas son las que se integraron en la investigación, así como el considerar la metodología y las técnicas utilizadas, tomando en cuenta que la gestión financiera permite delimitar cómo estos conceptos afectan la rentabilidad y productividad del sector.

\section{Conclusiones}

Con la investigación se pudo corroborar que a pesar de que en las empresas industriales cubanas el uso de técnicas de gestión en los procesos administrativos aún es insuficiente, esto ha quedado reflejado en los niveles de beneficio en comparación con las potencialidades que tiene el sector y la proyección de los indicadores de resultados, aunque con la aplicación del procedimiento se contribuye a mejorar el sistema de información y con ello las decisiones relevantes.

El Modelo Costo-VolumenUtilidad es una herramienta de la Contabilidad de Gestión muy eficaz para la toma de decisiones y la proyección de los niveles de venta. El procedimiento propuesto permitió tomar decisiones encaminadas al mejoramiento de la comercialización en la línea de jugos naturales de la Empresa Industrial considerándose el análisis de indicadores que permitieron realizar proyecciones adecuadas.

La aplicación de los escenarios en la investigación corroboró el limitado proceso de planificación y uso de las capacidades de la línea 
de jugos naturales de la Empresa Industrial. Los resultados obtenidos pudieron corroborar que el uso de técnicas de gestión adecuadas permite el desarrollo del proceso de toma de decisiones administrativas incentivando al logro de los objetivos propuestos por la organización e incremento de los niveles de producción y beneficios asociados a estos, además a través de esta técnica se pueden planificar los resultados operativos y tomar las decisiones adecuadas para su alcance.

\section{Bibliografía}

Arnaiz, Y. (2012). Estrategia económica para elevar la eficiencia en instalaciones hoteleras. Editado Memorias X Conferencia Científica UNICA 2012. ISBN 959. 16-0173-5

Culqui, D. (2012). Diseño del cuadro de mando integral para mejorar la gestión administrativa de la empresa i.m.esco, de la ciudad de Ambato. Repositorio Universidad Técnica de Ambato.

Fontalvo, T., Vergara, J.C., y de la Hoz, E. (2012). Evaluación del impacto de los sistemas de gestión de la calidad en la liquidez y rentabilidad de las empresas de la zona industrial Vía 40. Revista Pensamiento y Gestión, 32, 3-25.

Gómez, J., López, E., González, M. B., y Rodríguez, E. (2012). Sistemas contables de gestión, Recursos comerciales y capital humano. Un análisis en el sector agroalimentario español. Agrocien- cia, 47(1), 87-99. (Indexada en JCR).

Herrscher, E. (2010). Contabilidad y Gestión. Un enfoque sistémico para la acción. Ediciones Macch. EcuRed en línea.

http://site.ebrary.com/lib/pucesp/detail.ac tion? doc ID $=10479418 \& \mathrm{p} 00=\mathrm{co}$ ntabilidad+costos

Pastrana, A. (2012). Contabilidad de costos. Argentina: El Cid Editor apuntes, ProQuest ebrary. http:// site.ebrary.com/lib/pucesp/detail. action? doc $I D=10608822 \& \mathrm{p} 00=$ contabilidad+costos

Pérez, P. y Aspiolea, A. (2011). Análisis marginal para la determinación del precio óptimo en istalaciones hoteleras de Polo turístico Jardines del Rey de la cayeria norte de Ciego de Ávila. Revista Sabiduría, un espacio para escribir. 5ta edición. ISSN 1390-5090.

Pérez, R. (2015). El costo objetivo, una herramienta de gestión para mejorar la eficiencia económica. Revista de la Universidad Privada de Tacna. Perú.

Polimeni, R., Fabozzi, F., Adelberg, A., y Kole, M. (2011). Contabilidad de costos. Conceptos y aplicaciones para la toma de decisiones (2a. ed.). Cuba: Editorial Félix Varela, ProQuest ebrary.

Rendón, Y. (2012). "El presupuesto por centros de actividad para instalaciones hoteleras del destino turístico Jardines del Rey. Ciego de Ávila”. X Conferencia Científica Internacional UNICA 2012. ISBN 959-16-0173-5.

Salgado, C. (2011). Tendencias de investigación en contabilidad de gestión en Iberoamérica, (1998- 
2008). Cuadernos de Contabilidad, 12(30), 273-305.

Sinisterra, V. (2011) Contabilidad de costos. Colombia: Ecoe Ediciones, ProQuest ebrary. http://site. ebrary.com/lib/pucesp/detail.acti on?docID $=10552740 \&$ p00 $=$ cont abilidad+costos

Suárez, J. y Rodríguez, F. (2011). Sistemas de Costos. En: Selección de Guías de Estudio: Contabilidad y Finanzas. Cuba: Editorial Universitaria, ProQuest ebrary. http://site. ebrary.com/lib/pucesp/detail.acti on?docID $=10472626 \&$ p00 $=$ cont abilidad+costos

Torres, S. (2010). Contabilidad de costos: Análisis para la toma de decisiones. Tercera Edición. México: McGrawHill.

Villalta, C. (2011). Las técnicas gerenciales tradicionales y su incidencia en la gestión administrativa de la empresa plasticaucho de la ciudad de Ambato. Repositorio Universidad Técnica de Ambato.

Recepción: 07-19- 2016 - Aceptación: 12-12- 2016. 